\title{
A brief overview of the use of proton beam radiotherapy for gastrointestinal cancers
}

\section{History of protons in medicine}

In 1946, almost 50 years after the discovery and implementation of X-rays for the treatment of cancer, Dr. Robert Wilson at Harvard University proposed that proton beams could have potential clinical benefit (1). The first cancer patient was treated with proton beam therapy (PBT) in 1954 at the Lawrence Berkeley Laboratory, a high energy physics research facility in California. Only seven years later, the Harvard Cyclotron Laboratory opened in Boston, Massachusetts in 1961 and treated more than 9,000 patients with PBT over the next 40 years. The bulk of this experience was in the treatment of inoperable, radioresistant tumors that were superficial and/or of simple geometry and amenable to treatment with a fixed horizontal beam, such as ocular melanoma, prostate cancer, and sarcoma (2,3). The first hospital-based PBT center opened in 1990 at Loma Linda University in California. The Paul Scherrer Institute in Switzerland opened in 1996 the first compact gantry capable of administering pencil beam scanning intensity modulated proton therapy (IMPT). In the United States, MD Anderson Cancer Center in Texas initiated treatment in 2008 with a rotating gantry capable of administering pencil beam scanning IMPT. This ushered in a new era of modern PBT facilities capable of treating large, complex targets at essentially any site in the body. In 2015, Washington University commenced treatment with the first commercially available single room, compact proton accelerator and gantry, reducing the initial cost of investment to open a PBT center. As of December 2018, there are 30 PBT centers in operation in the United States (21 multi-room facilities and 9 single room facilities) and 45 PBT centers in operation outside of the United States, mostly in Asia and Europe (4).

\section{Physical properties of photons and protons}

Photons (also called X-rays) represent an electromagnetic wave of energy lacking mass or charge. Photons for medical use are generated either via natural radioactive decay referred to as gamma-rays (most commonly using Radium, Cesium, Cobalt, Iridium, or Iodine sources) or by accelerating and steering electrons into a tungsten target to produce high energy photons. The latter forms the basis for the modern linear accelerator, currently used to deliver external beam radiotherapy (RT) in most RT departments around the world.

In contrast to photons, protons have a discrete mass and a positive charge, and they reside in the nucleus of all atoms. Because the mass of a proton is roughly 2,000 times greater than that of an electron, the equipment required to accelerate and steer proton beams is substantially larger than that required for electrons to produce photons.

The physical interactions of photon and proton beams in tissue differ substantially and provide the rational for PBT. After a small build-up zone, photon beams reach maximum dose deposition in the superficial tissue and then deposit additional dose along the entire beam path until exiting the body (Figure 1). Although photons are attenuated by matter along the beam path, non-target normal tissues are exposed to dose along the "entrance" and "exit" beam path both in front of and behind the target (Figure 2A). Compared to photons, proton beams deposit a lower dose in front of the target upon entering tissue, deliver the majority of dose near the end of the energy range in the "Bragg Peak", and deliver almost no "exit dose" beyond the target (Figures 1,2B). Therefore, PBT offers a dosimetric advantage over photon RT, in that for a given dose of RT to a target, PBT delivers a lower dose to non-target normal tissue. In particular, PBT reduces the integral dose to the body by eliminating much of the "low dose bath" that results from the cross-firing of multiple beams required to create dose conformity in the high dose regions of photon RT plans (Figure 2C,D).

\section{PBT for gastrointestinal cancers}

Treatment of cancers of the gastrointestinal tract frequently involves multimodality therapy, including RT, chemotherapy, 


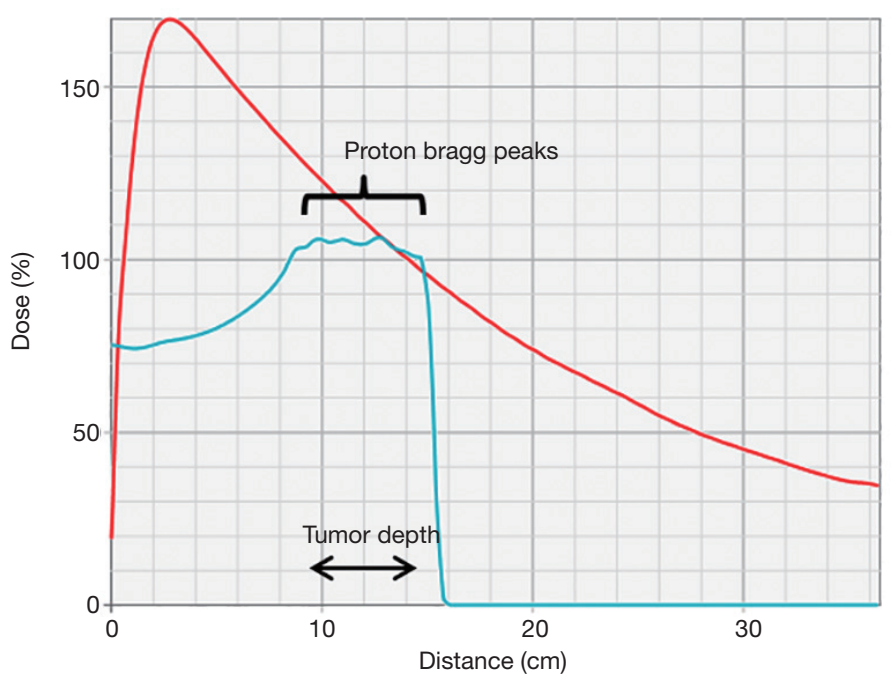

Figure 1 Dose delivered at depth for a single X-ray (red) or proton beam (blue). Note that the proton beam (versus the X-ray beam) delivers less dose proximal and no dose distal to the target.
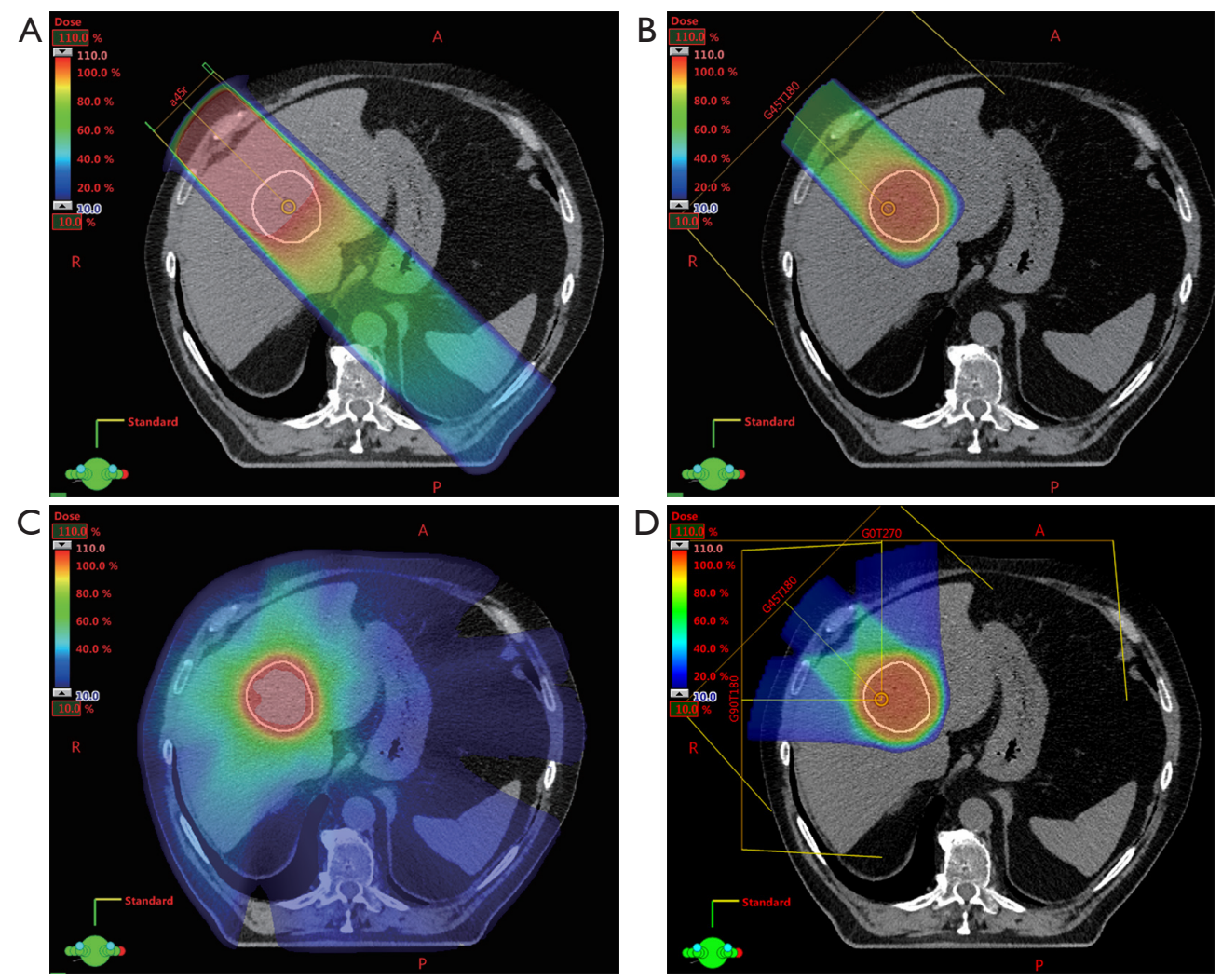

Figure 2 Dose distribution of single or multiple X-ray or proton beams for treatment of a liver tumor (white). The dose colorwash shows high (red), intermediate (green), and low (blue) doses. (A) Single X-ray beam; (B) single proton beam; (C) multiple X-ray beams; (D) multiple proton beams. 
and surgery. The use of concurrent chemoradiotherapy (CRT) compared to RT alone typically increases both the efficacy of treatment and the incidence and severity of acute and late toxicities. Independent of primary tumor site, common toxicities of CRT for gastrointestinal tumors may include fatigue, nausea, vomiting, anorexia, diarrhea, and bone marrow suppression. Additional toxicities are related to site of the primary tumor, including esophageal and cardiopulmonary toxicities for thoracic tumors, hepatic and renal toxicities for upper abdominal tumors, and genitourinary, skin, and skeletal toxicities for pelvic tumors. Importantly, the incidence and severity of CRT-related toxicity is directly related to dose and volume of normal tissue exposed to RT. For unresectable primary or recurrent tumors, RT dose escalation may improve tumor control, but toxicity from increased normal tissue RT exposure may negate potential benefits. Receipt of a previous course of RT also limits the use of a second course of RT due to risks of increased toxicity.

Based on the favorable dose distribution with superior normal tissue sparing offered by proton versus photon beams, PBT has potential clinical advantages for treatment of gastrointestinal cancers. There is potential to significantly limit radiation exposure to the uninvolved heart, lungs, liver, stomach and bowel. Limited early experience with the use of PBT for anal and rectal cancers was reported by investigators at Massachusetts General Hospital as early as 1977 (2,3). In 1988, investigators at the University of Tsukuba initiated a series of treatment protocols using high dose PBT for unresectable hepatocellular carcinoma $(5,6)$. These early experiences utilized passive scatter PBT delivery, had limitations in treatment angles and field size, and lacked sophisticated treatment planning and image guidance. Over the past 30 years, there have been substantial technologic advances in hardware and software, improving essentially all aspects of PBT treatment planning and delivery. With modern PBT equipment including rotating gantries and pencil beam scanning IMPT, tumors of almost any size, shape, and location within the gastrointestinal tract can be treated. Preliminary retrospective data support the safety and efficacy of pencil beam scanning IMPT for the combined modality treatment of esophageal, pancreatic, and anal cancers (7-9).

\section{Ongoing and future research}

To date, several single arm prospective studies have been completed for use of passive scatter PBT for hepatobiliary malignancies. Ongoing prospective phase I/II studies are evaluating the safety and feasibility of therapy escalation using PBT. National Cancer Institute (NCI) sponsored phase III randomized controlled trials are comparing proton $v s$. photon radiation for the treatment of esophageal cancer (NCT03801876) and hepatocellular carcinoma (NCT03186898). Further hardware and software advances are needed to improve upon treatment planning and delivery. Challenges remain, including characterizing and managing uncertainties in PBT dose distribution related to range uncertainties, variation in tissue densities, and organ motion. Additionally, further refinement in treatment planning is needed to account for variable radiobiological effectiveness of PBT, especially in the context of IMPT. Treatment with heavier charged particles such as carbon ions may offer additional biological advantages beyond PBT. Ongoing research efforts will further define the role of charged particle therapy for treatment of gastrointestinal malignancies.

In summary, PBT has emerged as a standard external beam RT modality with potential advantages over conventional photon therapy for the treatment of some patients with gastrointestinal cancers. In this special issue of the Journal of Gastrointestinal Oncology, we will provide a comprehensive review of the rationale, clinical data, current indications, and future directions for the use of PBT for gastrointestinal cancers.

\section{Acknowledgments}

None.

\section{Footnote}

Conflicts of Interest: The authors have no conflicts of interest to declare.

Ethical Statement: The authors are accountable for all aspects of the work in ensuring that questions related to the accuracy or integrity of any part of the work are appropriately investigated and resolved. 


\section{References}

1. Wilson RR. Radiological use of fast protons. Radiology 1946;47:487-91.

2. Suit HD, Goitein M, Tepper JE, et al. Clinical experience and expectation with protons and heavy ions. Int J Radiat Oncol Biol Phys 1977;3:115-25.

3. Suit H, Goitein M, Munzenrider J, et al. Evaluation of the clinical applicability of proton beams in definitive fractionated radiation therapy. Int J Radiat Oncol Biol Phys 1982;8:2199-205.

4. Particle Therapy Co-Operative Group - Particle therapy facilities in clinical operation. Available online: https://www.ptcog.ch/. Accessed December 312018.

5. Tanaka N, Matsuzaki Y, Chuganji Y, et al. Proton irradiation for hepatocellular carcinoma. Lancet 1992;340:1358.

6. Matsuzaki Y, Osuga T, Saito Y, et al. A new, effective, and safe therapeutic option using proton irradiation for hepatocellular carcinoma. Gastroenterology 1994;106:1032-41.

7. Prayongrat $\mathrm{A}, \mathrm{Xu} \mathrm{C}, \mathrm{Li} \mathrm{H}$, et al. Clinical outcomes of intensity modulated proton therapy and concurrent chemotherapy in esophageal carcinoma: a single institutional experience. Adv Radiat Oncol 2017;2:301-7.

8. Jethwa KR, Tryggestad EJ, Whitaker TJ, et al. Initial experience with intensity modulated proton therapy for intact, clinically localized pancreas cancer: Clinical implementation, dosimetric analysis, acute treatment-related adverse events, and patientreported outcomes. Adv Radiat Oncol 2018;3:314-21.

9. Wo JY, Plastaras JP, Metz JM, et al. Pencil Beam Scanning Proton Beam Chemoradiotherapy with 5-Fluorouracil and Mitomycin-C for Definitive Treatment of Carcinoma of the Anal Canal: A Multi-institutional Pilot Feasibility Study. Int J Radiat Oncol Biol Phys 2019. [Epub ahead of print].

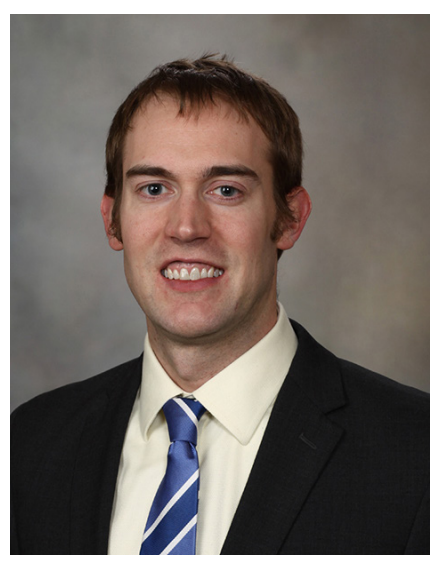

Christopher L. Hallemeier

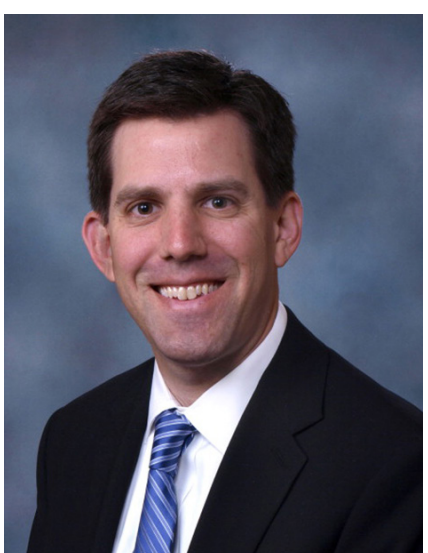

Jonathan B. Ashman

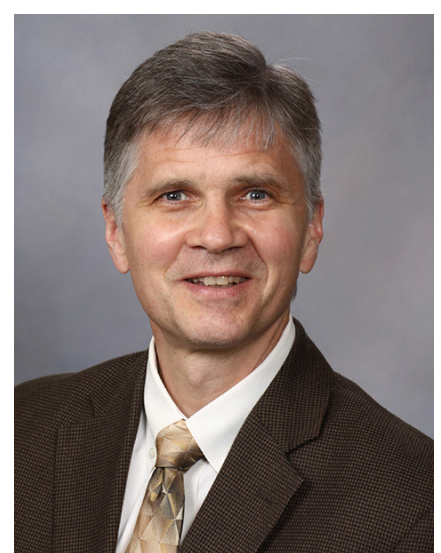

Michael G. Haddock 


\section{Christopher L. Hallemeier, MD}

Department of Radiation Oncology, Mayo Clinic, Rochester, MN, USA.

(Email: hallemeier.christopher@mayo.edu)

Jonathan B. Ashman, MD, PhD

Department of Radiation Oncology, Mayo Clinic, Phoenix, AZ, USA.

(Email:ashman.jonathan@mayo.edu)

Michael G. Haddock, MD

Department of Radiation Oncology, Mayo Clinic, Rochester, MN, USA.

(Email: haddock.michael@mayo.edu)

doi: 10.21037 /jgo.2019.07.06

View this article at: http://dx.doi.org/10.21037/jgo.2019.07.06

Cite this article as: Hallemeier CL, Ashman JB, Haddock MG. A brief overview of the use of proton beam radiotherapy for gastrointestinal cancers. J Gastrointest Oncol 2020;11(1):139143. doi: 10.21037 /jgo.2019.07.06 